\title{
Local Analysis for 3D Reconstruction of Specular Surfaces
}

\author{
Silvio Savarese \\ Pietro Perona \\ California Institute of Technology \\ Mail stop 136-93, Pasadena, CA 91125 \\ \{savarese,perona\}@vision.caltech.edu
}

\begin{abstract}
We explore the geometry linking the shape of a curved mirror surface to the distortions it produces on a scene it reflects. Our analysis is local and differential. We assume a simple calibrated scene composed of lines passing through a point. We demonstrate that local information about the geometry of the surface may be recovered up to the second order from either the orientation and curvature of the images of two intersecting lines, or from the orientation of the images of three or more intersecting lines. An explicit solution for calculating shape and position of spherical mirror surfaces is given.
\end{abstract}

\section{Introduction}

One of the main tasks of a visual systems is computing the shape of the objects [1]. A number of cues, notably stereoscopic disparity, texture gradient, motion parallax, contours and shading, have been shown to carry valuable information on surface shape, and have been studied extensively both computationally and psychophysically. The study of shading has been mostly restricted to the Lambertian case. Specularities have been mostly ignored with the significant exception of the analysis of specular highlights.

We are interested in exploring the possibility of recovering information on the shape of a surface from the specular component of its reflectance function. Since we wish to ignore the contributions of shading, we will study surfaces that are perfect mirrors. A curved mirror surface produces 'distorted' images of the surrounding world (see Fig. 1). For example, the image of a straight line reflected by a curved mirror is, in general, a curve. It is clear that such distortions are systematically related to the shape of the surface. Is it possible to invert this map, and recover the shape of the mirror from the images it reflects?

The general 'inverse mirror' problem is clearly underconstrained: by opportune manipulations of the surrounding world we may produce almost any image from any curved mirror surface as illustrated by the anamorphic images that were popular during the Renaissance. The pur-

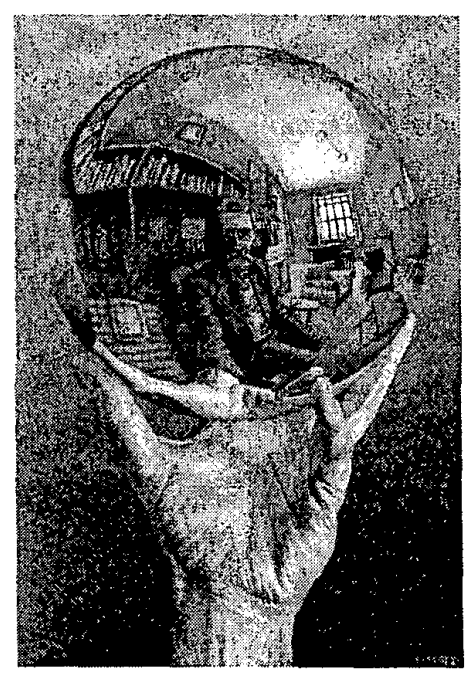

Figure 1. M.C. Escher (1935)

pose of this paper is to begin an investigation of the constraints of the problem, in order to explore the conditions under which the reconstruction might be feasible, possibly in conjunction with other visual cues. To this effect, we assume a calibrated world composed of the simplest primary structures: one point and one or more lines through it, and we study the relationship between the local geometry of the surface around the point of reflection, and the position, orientation and curvature of the reflected images of such point and lines.

Applications for our work include recovering the global shape of highly glossy surfaces. Two possible setup could be for istance: a) placing a suitable calibrated pattern of intersecting lines near the specular surface and applying our analysis at the locus of the observed reflections of the pattern intersections; b) placing a calibrated reference plane near the specular surface, projecting a suitable pattern with a calibrated LCD projector over the specular surface and ap- 
plying our analysis at the locus of the intersections reflected by the surface over the reference plane and observed by the camera; such setup is appealing since it requires the same hardware used by common structured lighting techniques. Finally, due to increasing interest in omniview cameras with curved surfaces mirrors, our work may provide useful mathematical tools for the analysis and the calibration of such imaging systems.

The main geometrical objects, constraints and notation are described in Sec. 2. The main geometrical techniques and results are obtained in Sec. 3 and Sec. 4. In Sec. 5 we discuss our findings and propose a number of issues for further research.

\subsection{Previous Work}

Previous works have mainly used specularities (or highlights) as a cue to infer information about the geometry of a specular surface. Koenderink and van Doorn [11] qualitatively described how pattern of specularities change under viewer motion. This analysis was extended by Blake $e t$ al. and incorporated in a stereoscopic vision framework [3] [2]. Additionally, Zisserman et al. [14] investigated what geometrical information can be obtained from tracked motion of specularities. Other approaches were based on mathematical models of specular reflections (e.g. reflectance maps) [9] or extention of photometric stereo models [10]. Oren and Nayar developed in [12] an analysis on classification of real and virtual features and an algorithm recovering the 3D surface profiles traveled by virtual features. Zheng and Murata developed a system [13] where extended lights illuminate a rotating specular object whose surface is reconstructed by analyzing the motion of the highlight stripes. Finally, in [8], Halsead et al. proposed a reconstruction algorithm where a surface model is fitted to a set of normals by imaging a specularly reflective surface with a pattern of light. Their results were applied in the interactive visualization of the cornea.

\section{The basic geometry}

The basic geometric setup is depicted in Fig. 2. A calibrated pattern is reflected by a curved mirror and the reflection is observed by a calibrated camera. The pattern may be formed by either one point or one point and one line, or 2 (or more) intersecting lines. Different kind of patterns, like circles or non planar patterns, might be also considered. Our analysis can easily be extended accordingly.

\subsection{Notation and Definitions}

A point (or a vector) in the $3 \mathrm{D}$ space is expressed by a column 3-vector and is denoted by a bold letter (e.g. $\left.\mathbf{x}=(x y z)^{T}\right)$. A vector whose norm is 1 is denoted by a bold letter with hat (e.g $\hat{\mathbf{n}}$ ). A coordinate reference system

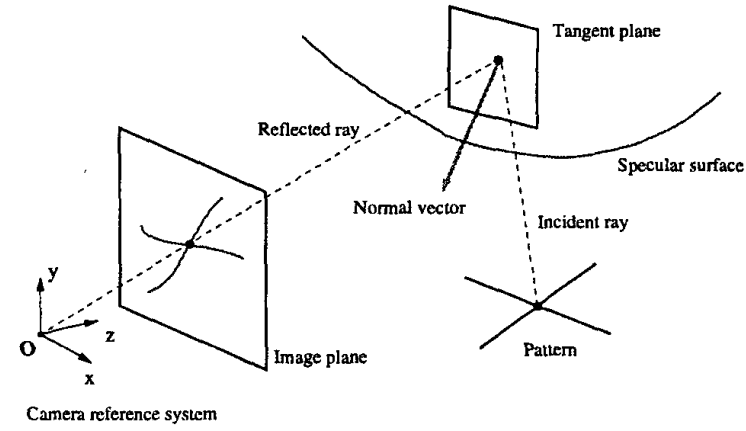

Figure 2. The basic setup.

$[X Y Z]$ is chosen with origin $\mathbf{O}_{c}$ in the center of projection of the camera. See Fig. 3. The lines of the pattern intersect in $\mathbf{x}_{p} . \mathbf{x}_{i}$ denotes the image of $\mathbf{x}_{p}$ reflected by the surface and $\mathbf{x}_{m}$ denotes the corresponding reflection point on the mirror surface. Since the camera and pattern are calibrated, $\mathbf{x}_{p}$ and $\mathbf{x}_{i}$ are known, whereas $\mathbf{x}_{m}$ is unknown. The normal to the surface in $\mathbf{x}_{m}$ is indicated by $\hat{\mathbf{n}}_{m}$ and is unknown as well. Let us call principal plane the plane defined by $\mathbf{x}_{i}, \mathbf{x}_{p}$ and $\mathbf{O}_{c}$ and its normal vector is $\hat{\mathbf{n}}_{p}$. Hence $\hat{\mathbf{n}}_{p}$ is a known quantity.

The geometry of our setup satisfies 3 basic constraints.

\subsection{Specular reflection constraints}

Constraint 1. This is the perspective projection constraint: the point $\mathbf{x}_{m}$ must belong to the line defined by $\mathbf{O}_{c}$ and $\mathbf{x}_{i}$, namely,

$$
\mathbf{x}_{m}=s \hat{\mathbf{x}}_{i}
$$

As a result, $\mathbf{x}_{m}$ is known up to a scalar $s$.

Constraint 2. This constraint accounts for the planar property of a specular reflection: the incident ray and the reflected ray must belong to the same plane, that is, the principal plane. Hence the normal vector $\hat{\mathbf{n}}_{m}$ to the surface at $\mathbf{x}_{m}$ belongs to the principal plane. Namely,

$$
\hat{\mathbf{n}}_{m}^{T} \hat{\mathbf{n}}_{p}=0
$$

Constraint 3. This constraint accounts for the reflection law: the angle between incident ray and normal to the point must be equal to angle between reflected ray and normal to point. Namely,

$$
\frac{\hat{\mathbf{n}}_{m}^{T} \mathbf{x}_{m}}{\left\|\mathbf{x}_{m}\right\|}=\frac{\hat{\mathbf{n}}_{m}^{T}\left(\mathbf{x}_{m}-\mathbf{x}_{p}\right)}{\left\|\mathbf{x}_{m}-\mathbf{x}_{p}\right\|}
$$

Such constraints can be combined in order to prove the following lemma:

Lemma 2.1 The normal vector $\hat{\mathbf{n}}_{m}$ is completely determined by $s$. 


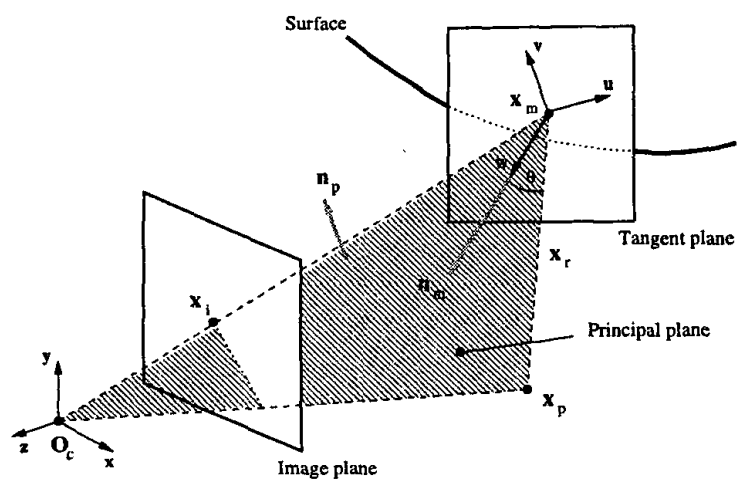

Figure 3. The geometry.

In fact, indicating $\frac{\left(\mathbf{x}_{m}-\mathbf{x}_{p}\right)}{\left\|\mathbf{x}_{m}-\mathbf{x}_{p}\right\|}$ in Eq. 3 by $\hat{\mathbf{x}}_{r}$ and by using Eq. 1, Eq. 3 becomes:

$$
\hat{\mathbf{n}}_{m}^{T}\left(\hat{\mathbf{x}}_{i}-\hat{\mathbf{x}}_{r}\right)=0
$$

By combining Eq. 4 with Eq. 2, $\hat{\mathbf{n}}_{m}$ can be expressed as a function of $s$ :

$$
\begin{aligned}
\hat{\mathbf{n}}_{m}(s) & =\left[\hat{\mathbf{x}}_{i}-\hat{\mathbf{x}}_{r}(s)\right] \times \hat{\mathbf{n}}_{p} \\
& =\left[\hat{\mathbf{x}}_{i}-\frac{\left(s \hat{\mathbf{x}}_{i}-\mathbf{x}_{p}\right)}{\left\|s \hat{\mathbf{x}}_{i}-\mathbf{x}_{p}\right\|}\right] \times \hat{\mathbf{n}}_{p}
\end{aligned}
$$

As a corollary, the reflection angle $\theta$ is parametrized by $s$ as well:

$$
\cos \theta=\frac{\sqrt{2}}{2} \sqrt{\frac{s-\hat{\mathbf{x}}_{i} \mathbf{x}_{p}}{\left\|s \hat{\mathbf{x}}_{i}-\mathbf{x}_{p}\right\|}+1}
$$

\subsection{The principal reference system}

We introduce a different coordinate reference system $[U V W]$ called the principal reference system. See Fig. 3. Such reference frame was introduced the first time by Blake in [2]. The principal reference system is centered in $x_{m}$; the $\hat{\mathbf{w}}$ axis is coincident with $\hat{\mathbf{n}}_{m}(s)$; the $\hat{\mathbf{v}}$ axis is coincident with $\hat{\mathbf{n}}_{p}$; the $\hat{\mathbf{u}}$ axis is given by $\hat{\mathbf{u}}=\hat{\mathbf{v}} \times \hat{\mathbf{w}}$. Thus, a point $\mathbf{x}$ in the $[X Y Z]$ and the corresponding point $\mathbf{x}^{\prime}$ in $[U V W]$ are related by transformation $\mathbf{x}^{\prime}=\mathbf{R}^{T}(\mathbf{x}-\mathbf{T})$, where $\mathbf{R}(s)$ $=\left[\begin{array}{lll}\hat{\mathbf{n}}_{p} \times \hat{\mathbf{n}}_{m}(s) & \hat{\mathbf{n}}_{p} & \hat{\mathbf{n}}_{m}(s)\end{array}\right]$ and $\mathbf{T}(s)=s \hat{\mathbf{x}}_{i}$. For instance, the center of the camera becomes $-\mathbf{R}(s)^{T} \mathbf{T}(s) ;$ Notice that the transformation is function of $s$. From now on, we shall always omit $s$ from the notation (unless we need to explicitly show such dependency) and assume that we work in the principal reference system.

\subsection{Discussion}

As stated by Lemma 2.1, as $s$ varies, a family of reflected points $\mathbf{x}_{m}(s)$ and corresponding normal vectors $\hat{\mathbf{n}}_{m}(s)$ is

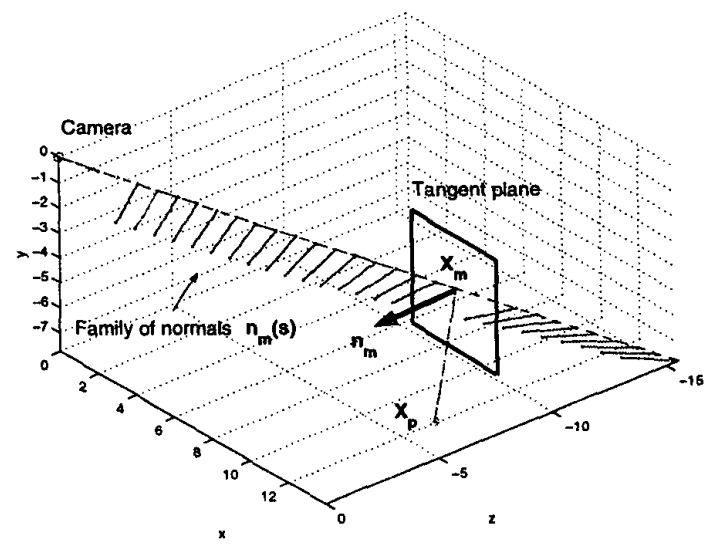

Figure 4. Family of normal vectors.

described. See Fig. 4. Each pair of points and corresponding normal is consistent with the observation $x_{i}$ of the reflected point. As a result, in order to find the parameter $s$ corresponding to the actual mirror surface, we need to introduce more constraints.

If the mirror surface is a plane, the surface is completely defined by $\mathbf{x}_{m}(s)$ and $\hat{\mathbf{n}}_{m}(s)$. Hence we obtain a family $\chi$ of the planes, parametrized by $s$, and consistent with our observation. As further constraint, we can use the correspondence between the orientation of one pattern line through $\mathbf{x}_{p}$ and the corresponding observed line reflected by the surface. Only one mirror plane within the family $\chi$ gives a reflected line which is consistent with the observation. Notice that such constraint does not carry meaningful information when either the pattern line belongs or is perpendicular to the principal plane. In fact, as we shall see qualitatively in Sec. 3.5, in both cases the corresponding reflected line is always consistent with the observation, no matter which plane within $\chi$ is considered.

If the surface is a sphere, we need to consider one more constraint. The tangent plane of the sphere in $x_{m}(s)$ is defined by $\hat{\mathbf{n}}_{m}(s)$ and therefore again fully described by $s$. However the pattern line reflected by the sphere is now imaged as a curved line. We shall show in Sec. 3 that the tangent to the imaged curve in $\mathbf{x}_{i}$ is not only function of the tangent plane but also function of the curvature $a$ of the sphere. Thus, the whole problem can be solved by jointly estimating both $s$ and $a$. This can be done by introducing a further constraint: for instance by relating one more pattern line through $\mathbf{x}_{p}$ and the corresponding reflected curve in the image line. We shall see in Sec 3.5 that a first order local analysis suffices to estimate both $s$ and $a$.

If a generic surface is considered, we can locally approximate the surface in $\mathbf{x}_{m}(s)$ with a paraboloid, which is fully described by the two parameters of curvature $a$ and $b$ and $a$ 
third parameter $c$ (tilt). Thus, we need to account for more constraints in order to solve for the new unknowns.

\section{First order local analysis}

In this section we examine what information can be retrieved from the image of the point and lines where up to first order information (point position and line tangents at the point) is considered.

\subsection{Parametric representation of the pattern}

As described in the previous section, the pattern can be formed, for instance, by intersecting two lines. Since the pattern is calibrated, we know the intersecting point location which can be redefined as $\mathbf{x}_{p o}$. The orientation of the line in space is also known. As a result, we can describe the generic pattern line in parametric form as follows:

$$
\mathbf{x}_{p}(t)=\mathbf{x}_{p o}+t \Delta \mathbf{p}
$$

where $\mathbf{x}_{p o}$ and $\Delta \mathbf{p}$ are known quantities.

\subsection{Mirror surface approximation}

In general, the mirror surface can be implicitly described by an equation $g(x, y, z)=0$. Since we are interested in analyzing the surface locally, we can always consider the corresponding Monge representation of the surface; that is, the surface can be described by the graph $z=G(x, y)$. In the principal reference system, the normal of the surface at the origin is $\hat{w}$ and the tangent plane to the surface at the origin is the plane defined by $\hat{\mathbf{u}}$ and $\hat{\mathbf{v}}$. Therefore the equation of the surface can be written in the special Monge form [7] as follows:

$$
\begin{aligned}
z & =\frac{1}{2 !}\left(a x^{2}+2 c x y+b y^{2}\right) \\
& +\frac{1}{3 !}\left(e x^{3}+3 f x^{2} y+3 g x y^{2}+h y^{3}\right)+\cdots
\end{aligned}
$$

For instance if the surface is locally a sphere of radius $r$, the graph is $z=r-\sqrt{r^{2}-x^{2}-y^{2}}$ and the Monge form is:

$$
z=\frac{1}{2 r}\left(x^{2}+y^{2}\right)+\frac{1}{8 r^{3}}\left(x^{4}+2 x^{2} y^{2}+y^{4}\right)+\cdots
$$

The surface can always be locally approximated by a paraboloid, provided that third and higher terms are negligible. Notice that the parameters $a, b$ and $c$ of the Monge form are unknown.

\subsection{First order differential analysis}

In this section we introduce the main mathematical tool to study the differential behavior of a specular reflection around the reflection point. In the same fashion as described by Chen and Arvo in [6] we define a path function $f$ which maps a point $x_{p}$ (within the pattern) into the corresponding reflection point $\mathbf{x}_{m}$ in the mirror surface, given a fixed observer $\mathbf{O}_{c}$. Since $\mathbf{x}_{p}$ is constrained to belong to the parametrized pattern line, the mapping can be expressed as follows:

$$
\mathbf{f}: t \in \Re \rightarrow \mathbf{x}_{m} \in \Re^{3}
$$

In other words, Eq. 10 defines a parametrized space curve lying within the mirror surface which describes the position of the reflection point $\mathbf{x}_{m}$, as $t$ varies.

We can use the Fermat Principle [4] of the shortest distance - the reflection point is a point that belongs to the mirror surface that locally minimizes the specular path length from the pattern to the observer - in order to solve $\mathbf{f}$ for $\mathbf{x}_{m}$. In other words, $\mathbf{x}_{m}$ can be found by minimizing the path length $d=\left\|\mathbf{x}_{m}-\mathbf{x}_{p}(t)\right\|+\left\|\mathbf{x}_{m}-\mathbf{O}_{c}\right\|$ and by imposing that $g\left(x_{m}\right)=0$, where $g(x)$ is the implicit function describing the mirror surface. At that end, by applying the Lagrange Multipliers Theorem, we obtain:

$$
\left\{\begin{array}{l}
-\frac{\left(\mathbf{x}_{m}-\mathbf{x}_{p}(t)\right)}{\left\|\mathbf{x}_{m}-\mathbf{x}_{p}(t)\right\|}-\frac{\left(\mathbf{x}_{m}-\mathbf{O}_{c}\right)}{\left\|\mathbf{x}_{m}-\mathbf{O}_{c}\right\|}+\lambda \nabla g(\mathbf{x})=0 \\
g(\mathbf{x})=0
\end{array}\right.
$$

which is a non linear system of 4 equations in 4 unknowns $\left(\mathbf{x}_{m}\right.$ and $\left.\lambda\right)$. Such a system can be denoted by $\mathbf{F}\left(t, \mathbf{x}_{m}, \lambda\right)=0$, where each component of $\mathbf{F}=$ $\left[\begin{array}{llll}F_{1} & F_{2} & F_{3} & F_{4}\end{array}\right]^{T}$ captures the left side of one of the equations in (11).

Unfortunately, there does not exist a closed form solution for $\mathbf{x}_{m}$, even for simple $g(\mathbf{x})$, like a sphere. However, if we call $x_{m_{o}}$ the solution for a given $t_{o}$, we can study the differential behavior of $\mathbf{f}(t)$ around $t_{o}$ by exploiting the Implicit Function Theorem. The theorem states that if $\left(t_{o}, \mathbf{x}_{m_{o}}, \lambda_{o}\right)$ is a solution for $F\left(t, x_{m}, \lambda\right)=0$ and if the $4 \times 4$ Jacobian matrix

$$
\mathbf{J}=\frac{\partial \mathbf{F}\left(t, \mathbf{x}_{m}, \lambda\right)}{\partial\left(\mathbf{x}_{m}, \lambda\right)}
$$

is not singular at $\left(t_{o}, \mathbf{x}_{m_{o}}, \lambda_{o}\right)$, then there exists an unique explicit function $\phi: t \rightarrow\left(\mathbf{x}_{m}, \lambda\right)$ that satisfies $\mathbf{F}(t, \phi(t))=$ 0 in a neighborhood of $\left(t_{o}, x_{m_{o}}, \lambda_{o}\right)$. Unfortunately the theorem does not tell us how to compute $\phi$. By differentiating $\mathbf{F}(t, \phi(t))$ in $\left(t_{o}, \mathbf{x}_{m_{o}}, \lambda_{o}\right)$, we achieve the following equation:

$$
\mathbf{B}=-\mathbf{J} \mathbf{M}
$$

where

$$
\begin{gathered}
\mathbf{B}=\frac{\partial \mathbf{F}\left(t, \mathbf{x}_{m}, \lambda\right)}{\partial t} \\
\mathbf{M}=\left[\begin{array}{llll}
\dot{u}(t) & \dot{v}(t) & \dot{w}(t) & \dot{\lambda}(t)
\end{array}\right]^{T}
\end{gathered}
$$

The dot indicates the derivative of the component with respect to $t$ and $\frac{\partial F_{i}}{\partial\left(\mathbf{x}_{m}, \lambda\right)}=\left[\frac{\partial F_{i}}{\partial u} \frac{\partial F_{i}}{\partial v} \frac{\partial F_{i}}{\partial w} \frac{\partial F_{i}}{\partial \lambda}\right]$, for $i=1, \cdots 4$. Eq. 13 essentially relates the tangent vector of the curve 
$\mathbf{f}(t)=[u(t) v(t) \quad w(t)]$ at $\mathbf{x}_{m_{\circ}}=\mathbf{f}\left(t_{o}\right)$ with the pattern geometry and the local parameters of the mirror surface.

In the principal reference system, $\mathbf{x}_{m_{\mathrm{o}}}$ is located at the origin. Furthermore we assume that, without loss of generality, $\mathbf{x}_{m_{o}}=\mathbf{f}\left(t_{o}\right)=0$ when $t_{o}=0$. We use the special Monge representation (Eq. 8) to describe our mirror surface. It is not difficult to see that, if we evaluate Eq. 13 at the reflection point $\mathbf{x}_{m_{\circ}}$, the $4^{\text {th }}$ row vector of $\mathbf{J}$ becomes $\left[\begin{array}{llll}0 & 0 & 1 & 0\end{array}\right]$, the $4^{\text {th }}$ column vector becomes $\left[\begin{array}{llll}0 & 0 & 1 & 0\end{array}\right]^{T}$ and $\frac{\partial F_{4}}{\partial \lambda}=0$. Furthermore the equation obtained by considering the $4^{t h}$ component of Eq. 13 implies that $\dot{w}\left(t_{o}\right)$ must be zero. We expect this result since the tangent of $\mathbf{f}(t)$ at $\mathbf{x}_{m_{o}}$ lies in the surface tangent plane, which is orthogonal to $\hat{\mathbf{w}}$.

As a result, Eq. 13 can be expressed in a more compact form:

$$
\left[\begin{array}{l}
\frac{\partial F_{1}}{\partial t} \\
\frac{\partial F_{2}}{\partial t}
\end{array}\right]=-\left[\begin{array}{ll}
\frac{\partial F_{1}}{\partial u} & \frac{\partial F_{1}}{\partial v} \\
\frac{\partial F_{2}}{\partial u} & \frac{\partial F_{2}}{\partial v}
\end{array}\right]\left[\begin{array}{c}
\dot{u}(t) \\
\dot{v}(t)
\end{array}\right]
$$

The entries of Eq. 16, valued at $\left(t_{o}, \mathbf{x}_{m_{o}}, \lambda_{o}\right)$, can be written as follows:

$$
\begin{aligned}
& \frac{\partial F_{1}}{\partial t}=\frac{1}{\left\|\mathbf{p}_{o}\right\|}\left(\Delta p_{w} \cos \theta \sin \theta-\Delta p_{u} \cos ^{2} \theta\right) \\
& \frac{\partial F_{2}}{\partial t}=-\frac{\Delta p_{v}}{\left\|\mathbf{P}_{o}\right\|} \\
& \frac{\partial F_{1}}{\partial u}=\cos ^{2} \theta \frac{s+\left\|\mathbf{p}_{o}\right\|}{s\left\|\mathbf{p}_{o}\right\|}-2 a \cos \theta \\
& \frac{\partial F_{2}}{\partial v}=\frac{s+\left\|\mathbf{p}_{o}\right\|}{s\left\|\mathbf{p}_{o}\right\|}-2 b \cos \theta \\
& \frac{\partial F_{1}}{\partial v}=\frac{\partial F_{2}}{\partial u}=-2 c \cos \theta
\end{aligned}
$$

where $s$ is the distance between $\mathbf{O}_{c}$ and $\mathbf{x}_{m_{\circ}}$ (see Eq. 1). Notice that the angle of reflection $\theta$ (see Eq. 6), $\left\|p_{o}\right\|$ and $\Delta \mathbf{p}=\left[\begin{array}{lll}\Delta p_{u} & \Delta p_{v} & \Delta p_{w}\end{array}\right]^{T}$ depend upon $s$.

The components of the tangent vector $\mathrm{t}=\left[\begin{array}{lll}\dot{u} & \dot{v} & \dot{w}\end{array}\right]^{T}$ of the curve $f(t)$ at $t_{o}$ can be derived from Eq. 16:

$$
\left[\begin{array}{c}
\dot{u}_{o} \\
\dot{v}_{o}
\end{array}\right]=\frac{1}{\Delta}\left[\begin{array}{cc}
J_{v}-2 b \cos \theta & 2 c \cos \theta \\
2 c \cos \theta & J_{u}-2 a \cos \theta
\end{array}\right]\left[\begin{array}{c}
B_{u} \\
B_{v}
\end{array}\right]
$$

where $\Delta=\left(J_{u}-2 a \cos \theta\right)\left(J_{v}-2 b \cos \theta\right)-(2 c \cos \theta)^{2}$, $B_{u}$ and $B_{v}$ indicate $\frac{\partial F_{1}}{\partial t}$ and $\frac{\partial F_{2}}{\partial t}$ respectively; finally $J_{u}$ and $J_{v}$ capture the remaining quantities within $\frac{\partial F_{1}}{\partial u}$ and $\frac{\partial F_{2}}{\partial v}$ respectively.

We remind here that such conclusion holds provided that mapping $\phi$ is unique in a neighborhood of $\left(t_{o}, \mathbf{x}_{m_{0}}, \lambda_{o}\right)$, namely, that $\mathbf{J}_{o}$ (i.e. $\mathbf{J}$ valued in $\left(t_{o}, \mathbf{x}_{m_{o}}, \lambda_{o}\right)$ ), is non singular. It is not difficult to show that the determinant of $\mathbf{J}_{o}$ is $-\Delta$. As a result, the condition $\Delta \neq 0$ is sufficient for the mapping $\phi$ to be unique.
Proposition 3.1 If the mirror surface is locally strictly convex in an neighborhood of $\mathbf{x}_{m_{o}}$, then $\Delta$ is always $\neq 0$.

PROOF. By hypothesis of locally strictly convex mirror in an neighborhood of $\mathbf{x}_{m_{0}}$ and recalling how the surface is located in the principal reference system, the second order parameters $(a, b$ and $c)$ of the expansion of Eq. 8 must satisfy:

$$
\left\{\begin{array}{l}
a<0 ; \quad b<0 \\
c^{2}<a b
\end{array}\right.
$$

Let us impose by contradiction that $\Delta=0$. Thus,

$$
c^{2}=\left(\frac{J_{u}}{2 \cos \theta}-a\right)\left(\frac{J_{v}}{2 \cos \theta}-b\right)
$$

Since $J u, J_{v}>0$ and the reflection angle $\theta$ must be $<\pi / 2$, Eq. 19 implies that at least one of the conditions in Eq. 18 cannot be verified.

From now on we shall always assume that $\Delta \neq 0$.

\subsection{Observation of the reflected pattern line}

As depicted in Fig. 2, the pattern line, reflected by the mirror surface, is imaged as a curve line in the image plane. We call such curve $\mathbf{f}_{i}$. $\mathbf{f}_{i}$ is essentially the perspective projection of $\mathbf{f}(t)$ into the image plane. Let us call $t_{o}$ the tangent vector of $f(t)$ at $x_{m_{0}}$ and $t_{i o}$ the tangent vector of $f_{i}$ at $\mathbf{x}_{i o}$, which is the perspective projection of $\mathbf{x}_{m_{o}}$ into the image plane. It is not difficult to show that $t_{i o}$ is parallel to the perspective projection of $t_{o}$ into the image plane. Furthermore $t_{i o}$ and $t_{o}$ are linked by the following relationship:

$$
\mathbf{t}_{o}=\hat{\mathbf{n}}_{m} \times\left(\hat{\mathbf{O}}_{c} \times \mathbf{t}_{i o}\right)
$$

In fact, since $t_{i o}$ is parallel to the perspective projection of $t_{o}$, the plane defined by $O_{c}$ and $t_{i o}$ contains $t_{o}$. Thus, if we call $\hat{\mathbf{n}}_{t}$ the normal vector to such a plane, $\hat{\mathbf{n}}_{t}^{T} \mathbf{t}_{o}=0$. Since $\hat{\mathbf{n}}_{m}^{T} \mathbf{t}_{o}=0, \mathbf{t}_{o}=\hat{\mathbf{n}}_{m} \times \hat{\mathbf{n}}_{t}$, which is Eq. 20 .

Additionally, we notice that all the terms in Eq. 17 do only depend upon the second order parameters (i.e. $a, b$ and c) of the expansion of Eq. 8, although no high order terms are assumed to be negligible in the Monge form describing the mirror surface. In other words, such result is valid no matter which surface is considered.

As a result, the following fundamental proposition holds:

Proposition 3.2 The tangent vector of the curve $\mathbf{f}_{i}$ at $\mathbf{x}_{i o}$ in the image plane only depends upon the geometry of the pattern line, the position of $\mathbf{x}_{m_{o}}$ and $\mathrm{O}_{c}$ and the second order parameters of the Taylor expansion of the surface Monge form.

In Fig. 5 an example is shown. The curve $f_{i}$ is generated by a sphere with curvature $a=-0.2$. The tangent $t_{\text {sphere }}$ of $\mathbf{f}_{i}$ at $\mathbf{x}_{i o}$ is depicted by the solid line. If the sphere is 


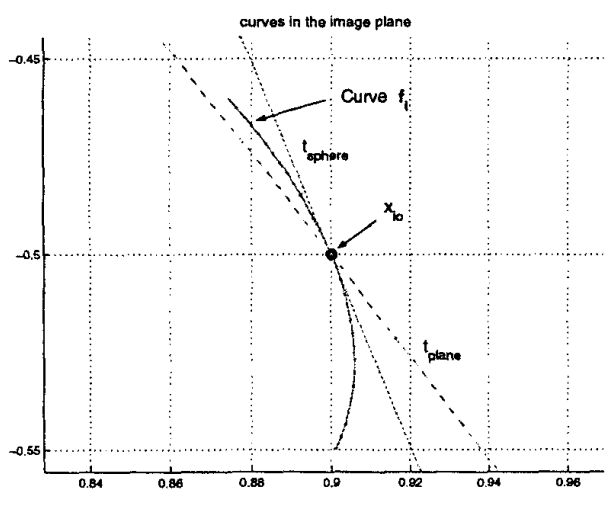

Figure 5. The tangent of the observed curve at $x_{i o}$ is function of the surface curvature.

replaced by a surface with zero curvature (namely, a plane coincident with the tangent plane of the sphere at $\mathbf{x}_{m_{0}}$ ), the new observed curve is a dashed straight line $t_{\text {plane }}$. Notice that $t_{\text {plane }}$ and $t_{\text {sphere }}$ have two different orientations.

\subsection{Surface recovery}

Let us consider Eq. 17. The unknowns are $s$ (embedded in $J_{u}, J_{v}, \theta, B_{u}, B_{v},\left\|\mathbf{p}_{o}\right\|$ and $\left.\Delta \mathbf{p}\right)$ and the second order parameters of the surface ( $a, b$ and $c$ ). Let $\varphi$ denote the angle between $\mathbf{t}_{o}$ and $\hat{\mathbf{u}}$. Thus, $\dot{u}_{o}=\left\|\mathbf{t}_{o}\right\| \cos \varphi$ and $\dot{v}_{o}=$ $\left\|t_{o}\right\| \sin \varphi$. The angle $\varphi$ can be estimated by using Eq. 20 and by measuring $t_{i o}$ in the image plane. Notice that $\left\|t_{o}\right\|$ is unknown as well.

By manipulating Eq. 17 we obtain:

$$
\tan \varphi=\frac{\left(J_{u}-2 a \cos \theta\right) B_{v}+2 c B_{u} \cos \theta}{\left(J_{v}-2 b \cos \theta\right) B_{u}+2 c B_{v} \cos \theta}
$$

We want to study this equation for different kinds of surfaces. If the mirror surface is a plane, then $a=b=c=0$. Thus, Eq. 21 becomes:

$$
\tan \varphi=\frac{B_{v} J_{u}}{B_{u} J_{v}}
$$

Since the only unknown is $s$, the constraint given by one pattern line is enough to solve the problem, as we have predicted in Sec. 2.4. If $s_{o}$ is the solution of Eq. 22, $\mathbf{x}_{m_{o}}=$ $s_{o} \mathbf{x}_{i_{o}}$ and the orientation of the plane in the $[X Y Z]$ reference system is completely determined (see Eq. 5).

If the mirror surface is locally a sphere, the corresponding Monge form is expressed by Eq. 9. In this case $a=b=$ $1 /(2 r)$ and $c=0$. Since we have one more unknown, we need to introduce one more constraint which can be given by a second pattern line. Let $\lambda_{1}$ and $\lambda_{2}$ be the 2 pattern lines. We obtain the following system of two equations:

$$
\left\{\begin{aligned}
\tan \varphi_{1} & =\frac{B_{v}^{1}\left(J_{u}-2 a \cos \theta\right)}{B_{u}^{1}\left(J_{v}-2 a \cos \theta\right)} \\
\tan \varphi_{2} & =\frac{B_{v}^{2}\left(J_{u}-2 a \cos \theta\right)}{B_{u}^{2}\left(J_{v}-2 a \cos \theta\right)}
\end{aligned}\right.
$$

where the superscript 1 and 2 indicate the quantities attached to $\lambda_{1}$ and $\lambda_{2}$ respectively. Thus

$$
\begin{gathered}
\Psi(s)=\tan \varphi_{1} B_{u}^{1} B_{v}^{2}-\tan \varphi_{2} B_{v}^{1} B_{u}^{2}=0 \\
a=\frac{B_{v}^{1} J_{u}-\tan \varphi_{1} B_{u}^{1} J_{v}}{2 \cos \theta\left(B_{v}^{1}-\tan \varphi_{1} B_{u}^{1}\right)}
\end{gathered}
$$

We can solve Eq. 23 for $s$. Once $s$ is known, we can compute $\mathbf{x}_{m_{\circ}}$ and $\hat{\mathbf{n}}_{m_{o}}$ in the $[X Y Z]$ reference system. The curvature of the sphere can be estimated by using Eq. 24 .

For more complex surfaces we have more unknowns ( $a$, $b$ and $c$ are in general $\neq 0$ ). We might think to constrain the problem with 3 pattern lines. For each line we can write a corresponding Eq 21 . We can arrange such equations in order to obtain a linear system in 3 equations in 3 unknowns $(a, b, c)$ parametrized by $s$. Numerical analysis shows that the rank attached to such linear system is $<3$ for $s=s_{o}$ whereas, in general, for $s \neq s_{o}$, is exactly 3 (where $s_{o}$ indicates the actual value of $s$ ). As a result, we can exploit such property to estimate $s_{o}$. On the other hand, this means that the local second order geometry of the surface cannot be fully retrieved. Namely, $a, b$ and $c$ can be estimated up to a scalar factor. The details of this analysis will appear in a forthcoming paper.

As anticipated in Sec. 2.4, we want to analyze two special cases where the reconstruction is not feasible: 1 ) pattern line lying in the principal plane $\pi_{p} ; 2$ ) pattern line orthogonal to $\pi_{p}$. Let us consider the first case. Since $\Delta p_{v}=0$, $B_{v}=0$. Thus, $\tan \varphi=2 c \cos \theta /\left(J_{v}-2 b \cos \theta\right)$. Notice that the value of $\varphi$ is the same no matter which line is considered within $\pi_{p}$. Furthermore, if the mirror surface is a plane or a sphere, $\varphi=0$, namely, the tangent vector always lies in $\pi_{p}$ for any $s$. The second case is similar. Since $\Delta p_{u}=\Delta p_{w}=0, B_{u}=0$. If the mirror surface is a plane or a sphere, $\varphi=\pi / 2$, namely, the tangent vector is always orthogonal to $\pi_{p}$ for any $s$. As a result, in both cases, the observed tangent vectors do not carry any useful information as far as the $s_{o}$ estimation is concerned.

\subsection{Numerical experiments}

In order to validate some of the theoretical results introduced in the previous section we have implemented a program to simulate specular reflections. Given the pattern point, a known surface (defined as a graph) and the observer, the routine computes the corresponding specular point over the surface by using a least square optimization technique to solve the system in Eq. 11. Thus, given a pattern line, 

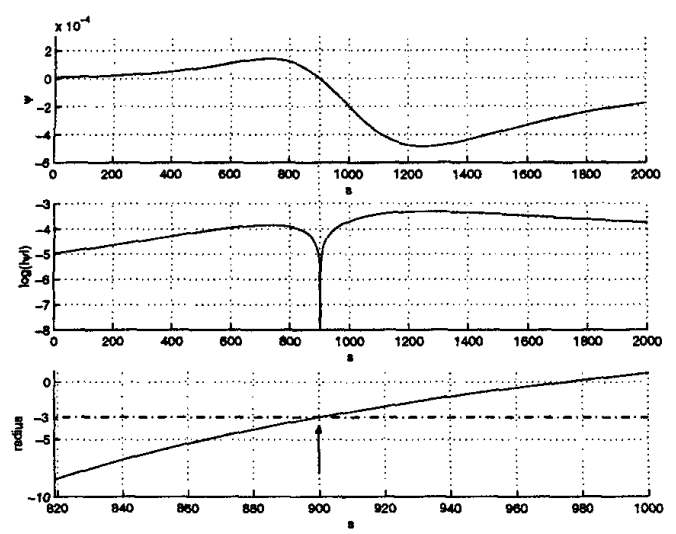

Figure 6. Estimation of $s$ and radius.

the routine computes the reflected curve imaged by the observer. Fig. 5 shows an example of curves imaged by the camera.

As an example of shape recovery, we validated Eq. 23 and Eq. 24 for the sphere. Given an image curve produced by the specular reflection simulator, the tangent lines at $\mathbf{x}_{i o}$ are estimated. Since location and orientation of the pattern lines are known, Eq. 23 can be solved for $s$ in the least square sense. Such value of $s$ can be used to estimate the curvature of the sphere by using Eq. 24. In Fig. 6 an example is shown. The sphere has radius $r=-3$ and the distance between observer and reflection point on the surface is given by $s_{o}=900$. The plots in the top and middle panel represents $\Psi$ and $\log (|\Psi|)$ respectively as function of $s$. The value of $s$ which minimizes $\log (|\Psi|)$ is the solution of Eq. 23. The minimum is achieved for $s=s_{o}=900$. Notice that the zero is easily detectable numerically since it is attached to the change in the sign of $\Psi$. The plot in the bottom panel represents the radius $r$ of the sphere as function of $s$ estimated by means of Eq. 24. As we can see, $r=-3$ at $s=900$.

Future work is needed to investigate from the theoretical point of view whether $\Psi(s)$ has a unique solution or not, although as result of our simulations there is no evidence of multiple solutions.

\section{Second order local analysis}

We now assume that we can measure the curvature $k$ of the lines in the image, as well as point position and line orientation. The second order analysis attached to the mapping $\mathbf{f}(t)$ can be obtained by taking the second derivative of $\mathbf{F}(t, \phi(t))$ in $\left(t_{o}, \mathbf{x}_{m_{o}}, \lambda_{o}\right)$. Thus, differentiating Eq 13, we obtain

$$
\frac{\partial \mathbf{B}}{\partial t}=-\frac{\partial \mathbf{J}}{\partial t} \mathbf{M}-\mathbf{J} \frac{\partial \mathbf{M}}{\partial t}
$$

where

$$
\frac{\partial \mathbf{M}}{\partial t}=\left[\begin{array}{llll}
\ddot{u}(t) & \ddot{v}(t) & \ddot{w}(t) & \ddot{\lambda}(t)
\end{array}\right]^{T}
$$

Eq. 25 and Eq.26 relate the second derivative of the curve $\mathbf{f}(t)$ at $\mathbf{x}_{m_{0}}$ to the pattern geometry and the local parameters of the surface. It is not difficult to show that each entry of Eq.25 may depend on up to the third order terms of the Taylor expansion for the surface Monge form (Eq. 8). Hence, whenever we use a paraboloid to approximate the surface, it is necessary to verify that the third order terms are negligible.

Let $k_{o}$ be the curvature of $\mathbf{f}(t)$ at $\mathbf{x}_{m_{o}}$. The general expression of the curvature is (see [5])

$$
k=\frac{\|\dot{\mathbf{f}}(t) \times \ddot{\mathbf{f}}(t)\|}{\|\dot{\mathbf{f}}(t)\|^{3}}
$$

Let $\hat{\mathbf{n}}_{f_{o}}$ be the principal normal of $\mathbf{f}(t)$ at $\mathbf{x}_{m_{0}}$. Notice that $\hat{\mathbf{n}}_{f_{0}}$ is not necessarily coincident with the surface normal $\hat{\mathbf{n}}_{m_{\mathrm{o}}}$. Let $\hat{\mathbf{b}}_{m_{\mathrm{o}}}$ be the normal to the osculating plane at $\mathbf{x}_{m_{\mathrm{o}}}$. The curvature $k_{o}$ can be related to the curvature $k_{i o}$ of the image curve $\mathbf{f}_{i o}$ at $\mathbf{x}_{m_{0}}$ by means of (see [7])

$$
k_{i o}=\frac{s k_{o} \mathbf{O}_{c}^{T} \hat{\mathbf{b}}_{m_{o}}}{\left(1-\left(\mathbf{O}_{c}^{T} \hat{\mathbf{t}}_{m_{o}}\right)^{2}\right)^{\frac{3}{2}}}
$$

where $\mathbf{O}_{c}$ and $\hat{\mathbf{t}}_{m_{o}}$ are function of $s, k_{i o}$ can be estimated within the image plane ${ }^{1}, \hat{\mathbf{b}}_{m_{\circ}}$ and $k_{o}$ are unknown. If $\alpha$ is the angle between $\hat{\mathbf{n}}_{f_{0}}$ and $\hat{\mathbf{n}}_{m_{\circ}}=\hat{\mathbf{w}}$, then

Lemma $4.1 \hat{\mathbf{n}}_{f_{o}}$ and $\hat{\mathbf{b}}_{m_{o}}$ are completely determined by $\alpha$ and $\hat{\mathbf{t}}_{m_{0}}$.

PROOF. Since $\hat{\mathbf{b}}_{m_{o}}$ is orthogonal to $\hat{\mathbf{n}}_{f_{o}}$ and $\hat{\mathbf{t}}_{m_{o}}$, the vectors $\hat{\mathbf{n}}_{f_{o}}, \hat{\mathbf{b}}_{m_{\circ}}$ and $\hat{\mathbf{w}}$ span a plane orthogonal to $\hat{\mathbf{t}}_{m_{\circ}}$.

Finally, we introduce the following proposition:

Proposition 4.1 The curvature of the curve $f(t)$ at $x_{m_{0}}$ can be expressed as a function of the observed curvature $k_{i o}, s$ and the second order terms of the Taylor expansion of the surface Monge form (namely, $a, b$ and $c$ ).

PROOF. By inspection, from the $4^{\text {th }}$ row of the vector equation (25), we obtain:

$$
a \dot{u}_{o}^{2}+2 c \dot{u}_{o} \dot{v}_{o}+b \dot{v}_{o}^{2}+\ddot{w}_{o}=0
$$

where $\dot{u}_{o}=\left\|\mathbf{t}_{o}\right\| \cos \varphi$ and $\dot{v}_{o}=\left\|\mathbf{t}_{o}\right\| \sin \varphi$. Furthermore, the second derivative of the curve $\mathbf{f}(t)$ at $\mathbf{x}_{m_{\circ}}$ is

$$
\ddot{\mathbf{f}}\left(t_{o}\right)=\left[\begin{array}{c}
\ddot{u}_{o} \\
\ddot{v}_{o} \\
\ddot{w}_{o}
\end{array}\right]=\frac{\partial\left\|\dot{\mathbf{f}}\left(t_{o}\right)\right\|}{\partial t} \hat{\mathbf{t}}_{m_{o}}+\left\|\dot{\mathbf{f}}\left(t_{o}\right)\right\|^{2} k_{o} \hat{\mathbf{n}}_{f_{o}}
$$

I The formula has been derived for the image sphere. Such quantities can be easily related to the image plane. 
The third component of Eq. 30 gives $\ddot{w}_{o}=\|\dot{\mathbf{f}}(t)\|^{2} k_{o} w_{f o}$, where $w_{f o}$ is the $w$ component of $\hat{\mathbf{n}}_{f_{o}}$. Notice that $w_{f o}=$ $\cos \alpha$. Thus, we can solve Eq. 29 for $k_{o}$, obtaining

$$
k_{o}=\frac{a \cos ^{2} \varphi+2 c \cos \varphi \cos \varphi+b \sin ^{2} \varphi}{\cos \alpha}
$$

By combining Eq. 31 and Eq. 28 and by Lemma 4.1, a relationship between $k_{o}, k_{i o}, a, b, c$ and $s$ can be written ${ }^{2}$.

\subsection{Surface Recovery}

If the surface is locally symmetric about $\mathbf{x}_{m_{\mathrm{o}}}$, the third order terms of the Taylor expansion of the surface Monge form are zero. Therefore Eq.25 allows us to relate $\left[\begin{array}{lll}\ddot{u}_{o} & \ddot{v}_{o} & \ddot{w}_{o}\end{array}\right]^{T}$ to $a, b, c$ and $s$. By combining the curvature measurements of 2 image curves at $\mathbf{x}_{i o}$, Eq.25, Eq.27, Eq. 28 and Eq.31, we introduce two more constraints which can be used, along with the first order ones, to estimate the unknowns $a, b, c$ and $s$. If the surface is not locally symmetric about $\mathbf{x}_{m_{o}}$, the third order terms are not necessarily zero. However if they are negligable in a neighborhood of $\mathbf{x}_{m_{o}}$, we can still estimate $a, b, c$ and $s$ as above.

\section{Summary and Conclusions}

We have proposed an analysis of the relationship between the image of a calibrated scene composed of lines through a point, and the geometry of a curved mirror surface on which the scene is reflected. Our analysis relates the position of a point in the image, and the orientation and curvature of lines through that point, to the local structure of the mirror (up to the third order) around the point of reflection.

Our main result is that it is possible to reconstruct the position and shape of the mirror surface up to the second order from the orientation and curvature of the images of two intersecting lines. Such reconstruction is local and is valid in a neighborhood where the third order terms may be neglected. Additionally, position and normal of the mirror surface may be obtained from the orientations of the images of three or more intersecting lines. A second order descrition of the surface can be retrieved up to a scale factor. The details of this analysis will appear in a forthcoming paper. An interesting result is that, even if only up to first order information is sought (i.e. the position of the reflection point and the tangent plane to the surface at such point) one cannot ignore the second order information in the surfaces. Explicit solutions for fully calculating the shape and position of planar and spherical mirror surfaces are given. The equations are validated by means of numerical simulations.

\footnotetext{
${ }^{2}$ Notice that the quantity at the numerator of Eq. 31 is the principal curvature of the surface in the direction of $\hat{t}_{m_{0}}$ and that Eq. 31 itself is an expression of the Meusnier's Theorem.
}

Future work is needed to study how sensitive the estimated parameters are with respect to noise added to the tangents and curvature estimates and validate our results with experiments with images of real specular surfaces.

We view our results as a promising start in the quest of computing the global shape of specular surfaces under fairly general conditions. The more interesting case of an uncalibrated world appears much more challenging and will require most certainly the integration of additional cues and some form of prior knowledge on the likely statistics of the scene geometry.

\section{Acknowledgments}

This work is supported by the NSF Engineering Research Center for Neuromorphic Systems Engineering (CNSE) at Caltech (EEC-9402726). We wish to thank Gabriel Taubin, Jean Ponce and Marzia Polito for helpful feedback and comments. We also wish to thank Matthew Cook and Massimo Franceschetti for many fruitful discussions as well as Rob Fergus for the review of the manuscript.

\section{References}

[1] T. Binford. Inferring surfaces from images. Artificial Intelligence, 17:205-244, 1981.

[2] A. Blake. Specular stereo. In IJCAI, pages 973-976, 1985.

[3] A. Blake and G. Brelstaff. Geometry from specularities. In Proc. ICCV, pages 394-403, 1988.

[4] M. Born and E. Wolf. Principle of optics: Electromagnetic theory and propagation. OPergamon Press, 1965.

[5] M. D. Carmo. Differential geometry of curves and surfaces. Prentice-Hall, 1976.

[6] M. Chen and J. Arvo. Pertubation methods for interactive specular refletions. IEEE Transactions on Visualization and Computer Graphics, 6:253-264, 2000.

[7] R. Cipolla and P. Giblin. Visual motion of curves and surfaces. Cambridge University Press, 2000.

[8] M. Halsead, A. Barsky, S. Klein, and R. Mandell. Reconstructing curved surfaces from specular reflection patterns using spline surface fitting normals. SIGGRAPH, 1996.

[9] G. Healey and T. Binford. Local shape from specularity. Computer Vision, Graphics, and Image Processing, 42(1):62-86, 1988.

[10] K. Ikeuchi. Determining surface orientation of specular surfaces by using the photometric stereo method. IEEE PAMI, 3:661-669, 1981.

[11] J. Koenderink and A. van Doorn. Photometric invariants related to solid shape. Optica Apta, 27:981-996, 1980.

[12] M. Oren and S. K.Nayar. A theory of specular surface geometry. Trans. IJCV, 2:105-124, Sept. 1997.

[13] J. Zheng and A. Murata. Acquiring a complete $3 \mathrm{~d}$ model from specular motion under the illumination of circularshaped light sources. IEEE PAMI, 8:913-920, 2000.

[14] A. Zisserman, P. Giblin, and A. Blake. The information available to a moving observer from specularities. Image and Video Computing, 7:38-42, 1989. 